\title{
Desenvolvimento de uma linha de cuidados para o idoso: hierarquização da atenção baseada na capacidade funcional
}

\author{
Development of a line of care for the elderly: hierarchical attention based on functional capacity
}

Resumo

O propósito deste artigo de atualização é contribuir para a estruturação de uma linha completa de cuidados para os idosos. Trata-se de um modelo baseado na identificação precoce dos riscos de fragilização dos usuários. Uma vez identificado o risco, a prioridade é a reabilitação precoce, a fim de reduzir o impacto das condições crônicas na funcionalidade. Partindo do pressuposto de que é adequado hierarquizar o processo de cuidado segundo as perdas funcionais que ocorrem com o passar dos anos, são estabelecidos sete pontos de atenção. O modelo é hierarquizado pela capacidade funcional, mas poliárquico na prática, pois a partir dos três primeiros níveis é possível seguir para qualquer outro ponto de atenção. Este modelo é vantajoso para o idoso, que amplia sua vida com qualidade; para a família, que terá seu familiar ativo e participativo; e para as operadoras de saúde, que evitarão internações repetidas e de alto custo.

\section{Abstract}

This paper aims to update knowledge about the structure of a complete line of care for the elderly. It is a model based on early identification of risks for frailty of users. Once identified the risk, priority is early rehabilitation in order to reduce the impact of chronic conditions on functionality. Assuming it is appropriate to prioritize the process of care, according to the functional losses that occur over the years, seven points of attention are established. The model is hierarchical by functional capacity, but polyarchical in practice, since from the first three levels it is possible to follow any other point of attention. This model is advantageous for the elderly, which improves the quality of life; for the family, whixh will have their relative activated and participatory, and for health operators, which will avoid repeated and costly hospitalizations.

\footnotetext{
Universidade Aberta da Terceira Idade. Universidade do Estado do Rio de Janeiro. Rio de Janeiro, RJ, Brasil.

2 Departamento de Odontologia, Centro de Ciências da Saúde. Universidade Federal do Rio Grande do Norte. Natal, RN, Brasil

Correspondência / Correspondence

Renato Peixoto Veras

UnATI-Universidade Aberta da Terceira Idade (UERJ)

Rua São Francisco Xavier, 524 - $10^{\circ}$ andar, bloco F

20559-900 Rio de Janeiro, RJ, Brasil

E-mail: unativeras@gmail.com
}

\section{Palavras-chave: Idoso.}

Prevenção de Doenças.

Cuidado Periódico. Qualidade de Vida. Linha de Cuidados.

Capacidade Funcional.

Key words: Elderly. Disease Prevention. Episode of Care. Quality of Life. Health Care Line. Functional Capacity. 


\section{INTRODUÇÃO}

Apesar de a longevidade da população brasileira ser considerada uma conquista, os sistemas de saúde são desafiados por esse fenômeno. Como acolher, assistir e tratar este novo contingente populacional, sem abandonar as gerações mais jovens e economicamente produtivas?

As ações e práticas de saúde oferecidas à população como um todo estão defasadas. Pertencem ao tempo em que o Brasil era um país de jovens. Há o reconhecimento de que o cuidado ao paciente com múltiplas doenças crônicas, que perduram por décadas, é extremamente custoso. Portanto, há necessidade de se buscar uma atenção eficiente para a população idosa.

O Grupo de Pesquisa Envelhecimento e Saúde, da UnATI-UERJ (Universidade Aberta da Terceira Idade, Universidade do Estado do Rio de Janeiro), pretende contribuir para uma nova lógica, que visa modificar o que atualmente é oferecido. O propósito deste artigo de atualização é apresentar o primeiro conjunto de reflexões que o grupo está realizando, de modo a contribuir para a estruturação de uma linha completa de cuidados para os idosos.

Este modelo teórico tem uma lógica pragmática, implicada na viabilidade de implantação e baseada em resultados mensuráveis. Tal lógica é aplicável a qualquer rede de atenção ao idoso, seja pública ou privada. No entanto, devido ao tamanho do Sistema Único de Saúde (SUS) e às imensas diferenças político-estruturais entre os municípios que o compõem, este modelo termina por se voltar para o setor privado, uma vez que, para implantá-lo, seria necessário dispor de uma agilidade que o setor público não tem.

O modelo é baseado na identificação precoce dos riscos de fragilização dos usuários, ou seja, busca-se intervir antes de o agravo ocorrer. Ou, pelo menos, o mais próximo possível, considerando-se que é viável fazer este tipo de intervenção em uma população cujo maior fator de risco é a própria idade. ${ }^{1}$ Pessoas que não desenvolverão alguma fragilidade são exceções nesse segmento populacional.
A identificação do risco e a integralidade da atenção nos diferentes pontos da rede são o cerne desta lógica. Uma vez identificado o risco, a prioridade é a reabilitação precoce, a fim de reduzir o impacto das condições crônicas na funcionalidade. A ideia é monitorar a saúde e não a doença; a intenção é postergar a doença, a fim de que o idoso possa usufruir seu tempo a mais de vida. A proposta é abandonar o paradigma baseado no gerenciamento de doença crônica e transformar a prática da atenção ao idoso segundo um novo paradigma fundamentado no gerenciamento de perfil de saúde dessa pessoa que, sendo idosa, provavelmente vive com alguma ou com um conjunto de patologias.

Partindo do pressuposto de que é adequado hierarquizar o processo de cuidado segundo as perdas funcionais que ocorrem frequentemente com o passar dos anos, são estabelecidos sete pontos de atenção. O modelo é hierarquizado pela capacidade funcional, mas poliárquico na prática, pois a partir dos três primeiros níveis é possível seguir para qualquer outro ponto de atenção.

Atualmente, os sistemas de saúde funcionam com poucos pontos de atenção e que não se articulam (ambulatório, hospital e instituições de longa permanência). Em geral, os pacientes entram nessa rede desarticulada num estágio muito avançado. A porta de entrada acaba sendo a emergência do hospital. Tal modelo, além de inadequado e anacrônico, tem uma péssima relação custo-benefício. Aliás, é por este motivo que os idosos não são bem-vindos pelas operadoras. No entanto, o problema é do modelo, centrado no hospital, com uso intensivo de tecnologias de alto custo, e seu fracasso não deve ser imputado aos clientes idosos. Há uma sobrecarga de usuários nos níveis de maior complexidade, pela carência de cuidado nos primeiros níveis. Na proposta ora apresentada, os primeiros três níveis são a base, e neles se concentra a imensa maioria da clientela. ${ }^{2-4}$

Deve-se frisar, no entanto, que o modelo explorará todas as fases de um processo evolutivo da fragilidade, estruturadas de modo hierárquico, desde o idoso saudável e ativo até o momento final da vida - em outras palavras, desde seu acolhimento e cadastramento no sistema até os cuidados paliativos na fase terminal (figura 1). 


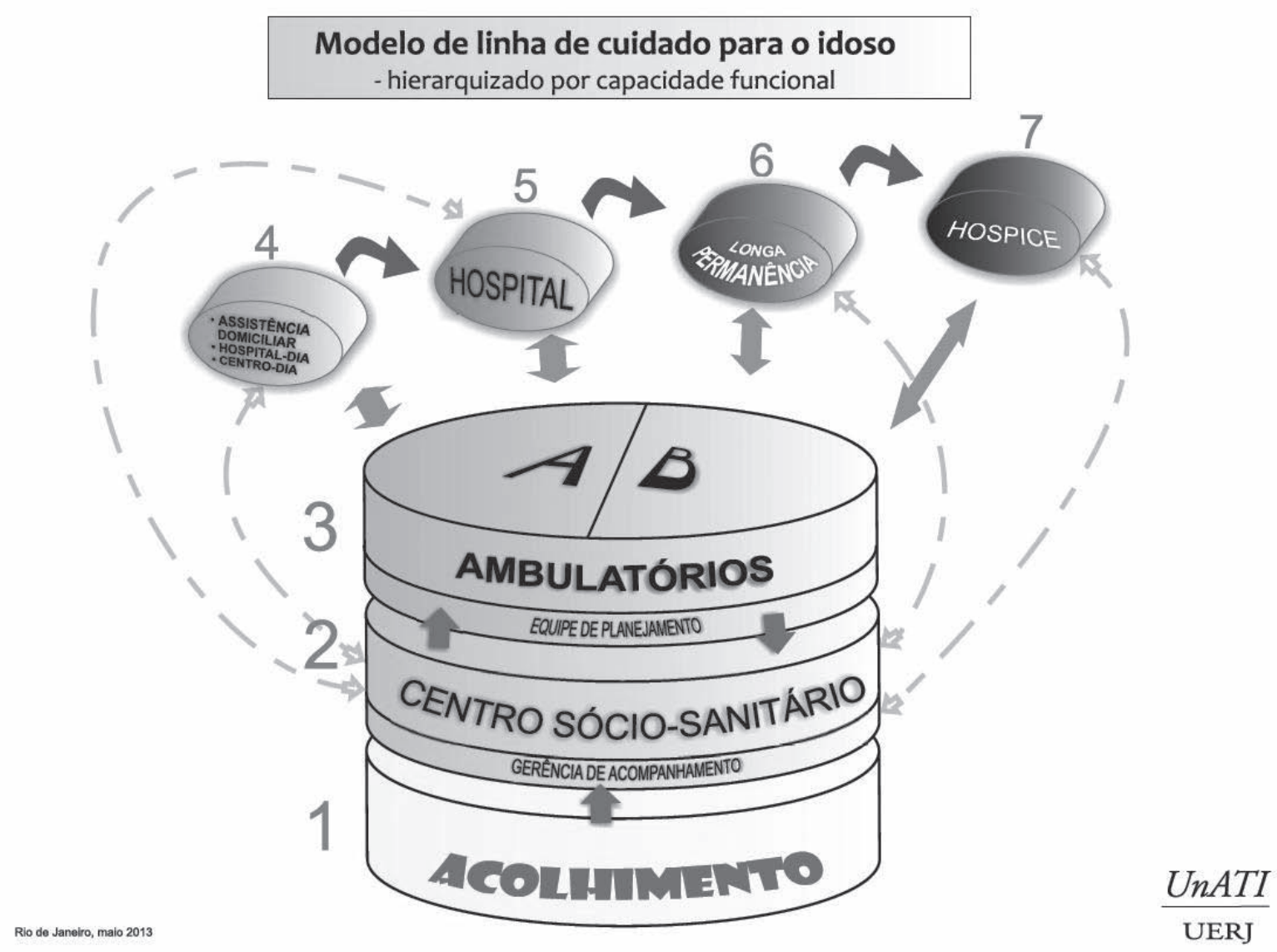

\section{DESCRIÇÃO DO MODELO TEÓRICO}

Ao se delinear um modelo que monitora continuamente o risco de fragilização, com instâncias intermediárias entre ambulatório e hospital, além de um nível especial para acolhimento, com uma abordagem educativa, é possível desacelerar o processo de fragilização e, como consequência, reduzir o custo da atenção à saúde e elevar a qualidade de vida do idoso. ${ }^{3}$ Além disso, tal abordagem é realizada de acordo com o nível de cuidados requeridos. Ou seja, a atenção será fornecida de acordo com a necessidade e no momento certo.

O modelo proposto é estruturado em 7 níveis, conforme apresentado na figura 1 . O nível 1 é o início da operação desta lógica e a base de entrada no sistema. Existem quatro aspectos que fundamentam o nível 1 do modelo: acolhimento, fidelização, integralidade e avaliação do risco de fragilização/incapacidade.
O nível 1 é o início da operação desta lógica e a base de entrada de todo o sistema.

Acolher a pessoa é fundamental para que ela possa desenvolver confiança no sistema e, assim, se fidelizar. A confiança e a fidelidade ao sistema são consequências da percepção do cliente do quanto ele é respeitado e do que lhe é oferecido. Adesão ao plano de cuidados é um fenômeno consequente à percepção do respeito e o resultado de um pacto estabelecido entre operadora e cliente. Esta pactuação se concretiza na forma de um contrato: as duas partes chegam a um acordo sobre como gerenciar a saúde do cliente, concordam e se comprometem. $\mathrm{O}$ acolhimento precisa ser educativo, para que o cliente tenha consciência de estar se comprometendo para cumprir sua parte no contrato. A tomada de decisão técnica sobre o encaminhamento do cliente precisa acontecer mediante esta pactuação e com base na avaliação do risco de incapacidade. A avaliação é específica para este segmento populacional. 
Operadora e usuário precisam acreditar que os procedimentos propostos são os melhores e mais adequados. Deve, portanto, trazer vantagens para ambos: isto é, o cliente, que percebe sua saúde bem cuidada e se sente protegido, e a operadora, que obtém a fidelização da clientela com custos mais baixos.

Em 2011, a Agência Nacional de Saúde (ANS) aprovou a Resolução Normativa $n^{\circ}$. 265/2011, que estabeleceu a opção por oferta da bonificação correspondente à adoção de programas de promoção do envelhecimento ativo voltados para todas as faixas etárias. O objetivo é melhorar a qualidade de vida/saúde de todos os beneficiários dos planos de saúde interessados em aderir ao programa. Portanto, neste modelo está previsto o desconto na mensalidade do plano de saúde concedido ao beneficiário como incentivo a sua participação.

Participar de programas de promoção e prevenção em saúde gera benefícios tanto para as operadoras quanto para os clientes. O estímulo financeiro para o engajamento dos usuários nos programas de prevenção tem se mostrado muito eficaz. Tais ações possuem uma lógica distributiva dos lucros, sendo a forma mais eficiente de adesão da clientela.

A educação do cliente envolve o cuidado de si. Os profissionais que integram o sistema precisam estar capacitados para respeitar o direito do cliente à autodeterminação. É importante que as limitações do plano sejam esclarecidas, para que o cliente assuma conscientemente o cuidado de si, sabendo que o agravamento de sua situação de saúde não é responsabilidade exclusiva do sistema. É uma responsabilidade compartilhada na sua capacidade de autodeterminação. Pequenas decisões que as pessoas tomam sobre seu próprio estilo de vida podem levar ao comprometimento de sua saúde. Os pontos-chave desse programa de educação do cliente, portanto, seriam o contrato pactuado, o esclarecimento dos direitos dentro do plano de saúde e o levantamento das necessidades de autocuidado, que seriam os deveres do contratante.

\section{DETALHAMENTO DO MODELO}

A UnATI/UERJ* tem larga experiência de atenção ao idoso e formação de recursos humanos, desenvolvidos ao longo de 20 anos. Com base nesta experiência e em uma abrangente revisão de literatura, foram identificados aspectos fundamentais dos mais importantes modelos de rede de atenção ao idoso e, desta forma, construiu-se esta proposta.

O primeiro aspecto é a existência de uma porta de entrada única - este é o nível 1. O segundo aspecto é a identificação do risco de fragilidade/incapacidade, para que seja criado um programa individualizado para cada cliente. Esta identificação de risco se inicia no nível 1, com uma triagem rápida, e continua no nível 2, com a avaliação funcional para início do processo de acompanhamento.

No nível 1 será efetuada uma triagem com instrumento breve (7 a 8 perguntas), com o objetivo de estabelecer o marco zero do perfil de risco do idoso. No nível 2, o idoso será recebido por seu gerente de acompanhamento, que a partir de rápida avaliação de risco do nível anterior, fará a avaliação funcional e então decidirá para que tipo de ambulatório ele irá, de acordo com a seguinte classificação de Moraes: ${ }^{6}$ idoso robusto e idoso em risco de fragilização.

O idoso robusto será acompanhado a partir do ambulatório no nível 3A e o idoso em risco de fragilização será acompanhado no ambulatório geriátrico, no nível 3B. O instrumento de triagem de risco será utilizado ao longo de todo o percurso assistencial do idoso, para a garantia de rastreamento contínuo de risco em todos os níveis. O gerente de acompanhamento, que passará a monitorar o idoso por todo o sistema a partir do nível 2, aplicará o instrumento de triagem de risco periodicamente.

O plano terapêutico engloba intervenções médicas, ações de educação, de promoção da

\footnotetext{
* Universidade Aberta da Terceira Idade / Universidade do Estado do Rio de Janeiro.
} 
saúde, de prevenção e de reabilitação, visando ao longo prazo. Embora o plano terapêutico seja estabelecido no nível 3, no nível 2 cada indivíduo é designado para um profissional que irá iniciar o acompanhamento do seu planejamento terapêutico (o gerente de acompanhamento).

Portanto, o nível 1 é o acolhimento do cliente imediatamente após a aquisição do plano de saúde. Neste nível, a prioridade é a ação educativa e o estabelecimento do marco zero do rastreamento contínuo de risco. Nesse momento, ele também é orientado sobre o programa, e o registro eletrônico de todo seu percurso assistencial é iniciado. A orientação sobre o programa inclui o esclarecimento sobre a forma de atendimento, a lógica das intervenções e as ações disponíveis. Neste espaço também estarão disponíveis materiais educativos para que, de acordo com a necessidade do cliente, já se possa iniciar a orientação sobre patologias crônicas, como hipertensão, diabetes, osteoartrite, uso seguro de medicação, nutrição saudável, segurança ambiental e atividade física, entre outras.

Esta abordagem educativa vem subsidiar a estratégia de educar o cliente, para que este possa entender sua responsabilidade no gerenciamento da própria saúde. O cliente precisa se inserir e participar ativamente do seu processo de promoção da saúde, porque essas ações dependem de uma mudança de atitude, que precisa ser feita a partir de uma pactuação com responsabilização do principal interessado, além do reforço da bonificação e premiação para aqueles que seguirem os programas. É importante ressaltar que tais programas de bonificação e premiação não são atrelados a resultados, apenas à participação do usuário. Um obeso participando de um programa de bonificação com atividade física, por exemplo, não precisa emagrecer para ter o benefício, basta participar.

Outra ação muito importante no acolhimento é a abertura do registro eletrônico de todo o percurso assistencial. É fundamental que exista um sistema de registro único e todo o processo terapêutico seja informatizado, para que haja continuidade em todos os níveis. Trata-se de um registro eletrônico único, longitudinal, multiprofissional, que acompanha o indivíduo desde o acolhimento, passando pelo centro de convivência (registro das atividades realizadas pelo cliente, bem como acompanhamento de seu perfil de saúde) e continuando por todo seu percurso assistencial. Todas as informações estarão disponíveis com acesso por cartão eletrônico.

Considerando a imperiosa necessidade de esse sistema ser resolutivo, se for identificado um alto risco na triagem, a pessoa é encaminhada para um nível de maior complexidade, imediatamente após a avaliação funcional realizada no nível 2 , retornando ao nível 3 após resolução da situação de risco. Por exemplo, se na avaliação foi identificada a necessidade de hospitalização, o idoso vai imediatamente para o hospital (nível 5). Após a alta, ele retorna ao nível 3 (ambulatório), que é o nível de tomada de decisão sobre o plano terapêutico. Portanto, embora esse indivíduo seja atendido em um nível mais avançado, suas informações serão captadas pela equipe de planejamento terapêutico do nível 3.

O nível 2 é um centro de atenção sóciosanitária, onde o idoso é atendido por seu gerente de acompanhamento, que realiza a avaliação funcional e passa a acompanhar seu percurso. Essa avaliação, denominada "avaliação funcional", inclui a avaliação do suporte social, da situação das doenças prévias e do atendimento recebido. $\mathrm{Na}$ entrevista serão levantadas as situações de risco tradicionalmente conhecidas em geriatria - cognição, humor, funcionalidade, mobilidade, uso de medicação, suporte social, funcionamento sensorial -, que levam à identificação de síndromes geriátricas.

Este nível se caracteriza por ser o ponto de atenção da rede que oferece suporte social, pois um suporte social frágil se configura também como risco. ${ }^{7}$ Visando minimizar esse risco, a unidade sócio-sanitária deverá incluir sistemas de apoio à vida autônoma e independente, a saber: alimentação (restaurante), serviços de 
suporte à família, call center com atendimento por gerontólogos, para todo o tipo de orientação, centro de convivência e conveniência, local onde se podem adquirir facilidades para os idosos - equipamentos variados, compra de pacotes de turismo, ingressos e transportes para lazer, como teatros e visitas a museus, ou seja, tudo que facilite a vida do idoso e seus familiares, serviços de reabilitação e de apoio ao cuidado e ao autocuidado.

Tais ações, vinculadas ao nível 2, são substitutivas para o suporte social, que visa facilitar uma vida ativa para aqueles autônomos e independentes, como também com facilidades de equipamentos de suporte para auxiliar as famílias com idosos dependentes. Além disso, há outros sistemas de apoio para auxiliar o autocuidado, incluindo a supervisão da administração de medicamentos e o agendamento de consultas. $\mathrm{O}$ nível 2 conta com ações que reforçam o suporte social do cliente onde for necessário.

É importante destacar que um idoso robusto não é um idoso que não tem doença. É o idoso que tem sua situação crônica controlada, sendo portanto um idoso autônomo e independente, ${ }^{6,7}$ ou seja, apresenta baixo risco. Este cliente será estimulado a participar das atividades que compõem o nível 2 - atividades de socialização, prevenção e promoção da saúde, suporte nutricional, fisioterapia, reabilitação e educação física - e será acompanhado pelo ambulatório do nível 3A.

O idoso que apresenta risco baixo e moderado será acompanhado ambulatorialmente. Dependendo da avaliação que for realizada no nível 2, será possível decidir se a intervenção necessária está no nível ambulatorial clínico (nível 3A) ou geriátrico (nível 3B). No nível $3 \mathrm{~A}$, o acompanhamento é realizado por equipe multiprofissional, voltando sua abordagem para a manutenção e reabilitação da funcionalidade. O ambulatório 3B é específico; é um ambulatório geriátrico, voltado para o idoso mais frágil ou de maior risco, que demanda intervenção por equipe interprofissional, com trabalho integrado, especializada em Geriatria.

Como se pode observar, os três primeiros níveis do modelo são repletos de ações e profissionais envolvidos. As ações intensivas são realizadas de modo a interromper/diminuir a evolução da fragilidade, paralelamente à redução dos custos, pois os gestores sabem que nos modelos de saúde atuais, o custo elevado se dá no hospital e nas unidades de tratamento intensivo, e não no pagamento de profissionais de saúde ou nos programas de prevenção ou monitoramento de saúde.

Outro aspecto central para o sucesso do modelo é que os pacientes são induzidos a fazerem parte dos programas e ações propostas, ao invés da lógica vigente de utilizar o plano apenas quando da realização de exames complexos ou da entrada em um hospital com doença em estágio avançado. Em síntese, nossa proposta é investir na saúde para se reduzir o gasto com a doença.

\section{CONCLUSÃO}

As despesas com cuidados voltados para doenças crônicas sobem em todo o mundo e ocupam proporções cada vez maiores nos orçamentos públicos e privados.

A tendência brasileira é que, nas próximas décadas, os gastos com saúde sofram aumento substancial e se transformem em um grande desafio fiscal. Existe ainda a expectativa de que outros fatores aumentem o número de idosos necessitados de cuidados, mesmo considerando a redução na proporção da severidade de doenças nessa faixa etária - ocorrida graças a avanços na prevenção de doenças e nas práticas assistenciais. Por fim, fatores de risco que em outras épocas alcançavam predominantemente o homem, como 
o consumo de álcool e tabaco ou o estresse no trabalho, passam a afetar também as mulheres. O resultado disso será a redução da diferença de expectativa de vida entre homens e mulheres, que hoje em dia é oito anos a mais para elas.

A magnitude do aumento dos gastos em saúde com a população idosa será determinante para o futuro: ou esses anos a mais serão vividos de forma saudável ou com enfermidades e dependência. A prevenção, a manutenção da saúde e da autonomia e o retardamento de doenças e fragilidades serão os maiores desafios relacionados à saúde, decorrentes do envelhecimento da população. Assim, qualquer política destinada aos idosos deve levar em conta a promoção do bem-estar e a manutenção da capacidade funcional.

Ocorre no Brasil um fato novo, em consonância com a perspectiva de preservar a saúde e propiciar mais anos de vida saudável: a Agência Nacional de Saúde (ANS) incentiva a participação de usuários de planos de saúde em programas de envelhecimento ativo, com a possibilidade de descontos nas mensalidades. A proposta tem impacto importante para a população de mais idade. A lógica do sistema de saúde está pautada no ciclo de tratamento das doenças e não da prevenção; inverter esse modelo é imperativo para se assegurar mais qualidade.

\section{REFERÊNCIAS}

1. Caldas CP, Veras RP, Motta LB, Lima KC, Kisse CBS, Trocado CVM, et al. Rastreamento do risco de perda funcional: uma estratégia fundamental para a organização da Rede de Atenção ao Idoso. Ciênc Saúde Coletiva [periódico na Internet]. 2013 [acesso em 29 maio 2013]. Disponível em: http://www. cienciaesaudecoletiva.com.br/artigos/artigo_int. php?id_artigo=12271.

2. Gragnolati M, Jorgensen $\mathrm{OH}$, Rocha R, Fruttero A. Growing old in an older brazil: implications of population aging on growth, poverty, public
A aplicação do aporte epidemiológico e a ênfase em prevenção fazem com que essa nova abordagem favoreça a redução dos custos assistenciais, em paralelo à ampliação da qualidade de vida, o que implica reorganizar os serviços de saúde. Qualquer política contemporânea para o setor deve valorizar o envelhecimento saudável, com manutenção e melhoria da capacidade funcional, prevenção de doenças e recuperação da saúde e das capacidades funcionais. Sem uma abordagem preventiva e integral, que associe reflexão epidemiológica e planejamento de ações de saúde, não há saída possível.

Em síntese, um modelo de atenção à saúde do idoso que se pretenda eficiente deve aplicar todos os níveis de cuidado: possuir um fluxo bem desenhado de ações educativas, promoção da saúde, prevenção e postergação de moléstia, assistência precoce e reabilitação de agravos. Essa linha de cuidados se inicia na captação e no monitoramento do idoso e somente se encerra nos momentos finais da vida, na unidade de cuidados paliativos.

Segundo o modelo ora proposto, todos podem se considerar vencedores: o idoso, que amplia sua vida com qualidade; a família, que terá um ente querido ativo e participativo; e as operadoras de saúde, que evitarão internações repetidas e de alto custo.

finance and service delivery. Washington: The World Bank; 2011.

3. Teisberg EO, Porter ME. Repensando a saúde: estratégias para melhorar a qualidade e reduzir os custos. Porto Alegre: Artmed, Bookman; 2006.

4. Porter ME. A strategy for health care reform: toward a value-based system. N Engl J Med 2009;361:109-12.

5. Brasil. Resolução Normativa n. 265, de 19 de agosto de 2011. Dispõe sobre a concessão de bonificação aos beneficiários de planos privados de assistência 
à saúde pela participação em programas para Promoção do Envelhecimento Ativo ao Longo do

Curso da Vida e de premiação pela participação em programas para População-Alvo Específica e programas para Gerenciamento de Crônicos. Diário Oficial da União, Poder Executivo, Brasília, DF, 22 ago 2011. Seção 1, p. 40.
6. Moraes EN. Atenção à saúde do idoso: aspectos conceituais. Brasília: Organização Pan-Americana da Saúde; 2012.

7. Moraes EN. Avaliação multidimensional do idoso: a consulta dos idosos e os instrumentos de rastreio. Belo Horizonte: Folium; 2010. 